\title{
45 Gbps Direct Modulation of Two-Section InP-on-Si DFB Laser Diodes
}

\author{
M. Shahin ${ }^{1,2}$, Student Member, IEEE , K. Ma ${ }^{3}$, A. Abbasi ${ }^{1,2}$, Member, IEEE, , G. Roelkens ${ }^{1,2}$, \\ Member, IEEE, and G. Morthier ${ }^{1,2}$, Senior Member, IEEE
}

\begin{abstract}
Two-section heterogeneously integrated InP-on-Si DFB laser diodes are demonstrated. In the modulation response, the relaxation oscillation frequency of $10 \mathrm{GHz}$ is followed by a second resonance to achieve nearly $25 \mathrm{GHz} 3-\mathrm{dB}$ modulation bandwidth and 45 Gbps NRZ-OOK transmission.
\end{abstract}

Index Terms - InP-on-Si, Distributed Feedback Lasers, Direct Modulation, Silicon Photonics, Semiconductor Laser Diodes, TwoSection Laser Diodes.

\section{INTRODUCTION}

$\mathrm{S}$ ilicon has been motivating many photonics researchers over the past decades to investigate active and passive optical components. Typically known as silicon photonics, the platform is attractive for two main reasons: a dense integration potential, and the possibility to use the mature CMOS electronics fabrication technology. The drawback of silicon lies in its indirect bandgap, making it challenging to be used for light sources. With the help of direct bandgap materials, such as III-V semiconductors, this drawback can be overcome. The heterogeneous integration of III-V semiconductors on silicon can be realized using die-to-wafer bonding [1]. In that case, the III-V material is bonded on silicon with the help of an adhesive material, typically divinylsiloxane-bis-benzocyclobutene (DVS-BCB), or using molecular bonding.

For many applications, DFB laser diodes are preferred as light sources due to their single mode behavior with large SideMode-Suppression-Ratio (SMSR). The fabrication of such lasers in the III-V-on-silicon platform is promising and has high potential for different applications. For example, for wavelength tuning, discrete wavelength tuning over $55 \mathrm{~nm}$ was demonstrated using sampled DFB gratings [2]. As for radioover-fiber applications, self-pulsating laser diodes with controlled frequency between 10 and $40 \mathrm{GHz}$ were demonstrated using two-section DFB laser diodes [3]. Finally, for datacom applications, high-speed DFB laser diodes were recently demonstrated at $56 \mathrm{Gbps}$ with direct modulation and 2 x 56 Gbps with electro-absorption modulation [4, 5]. A detailed report about other advances on this platform is given by [6].

Classical single section InP-on-Si DFB laser diodes are limited by their relaxation oscillation frequency. Fortunately, there are some advanced laser designs that improve the modulation bandwidth substantially. For example, an external cavity that exploits the Photon-Photon-Resonance (PPR) effect improved the modulation bandwidth from its relaxation oscillation frequency peak at $8 \mathrm{GHz}$, to a second resonance peak around $30 \mathrm{GHz}$ [4]. This improved the achieved 3-dB modulation bandwidth to $34 \mathrm{GHz}$. An alternative laser design that will be investigated in this paper is referred to as a twosection laser, or coupled cavity laser [7, 8]. Simulations show that such designs can enhance the modulation bandwidth to values as high as $50 \mathrm{GHz}$ due to the beating between the main mode and a closely-located side mode. An actual device utilizing this concept was fabricated and measured in [9], in which a monolithic InP DFB laser demonstrated a modulation speed of $112 \mathrm{Gbps}$. Here, the beating between the DBR and DFB modes, which are separated by $50 \mathrm{GHz}$, gives the modulation enhancement at $50 \mathrm{GHz}$.

In this paper, a two-section laser diode is demonstrated on the InP-on-Si platform. First, the design and fabrication are discussed. Then, a small signal modulation bandwidth of 25 $\mathrm{GHz}$ is demonstrated. Third, data transmission experiments are conducted, and transmission at $45 \mathrm{Gbps}$ is verified at bit error ratios that are substantially below the Forward-ErrorCorrection (FEC) threshold after propagation over $2 \mathrm{~km}$ of nonzero dispersion shifted fiber.

\section{DESIGN AND FABRICATION}

The laser structure was used previously to demonstrate selfpulsating laser diodes [3]. The two sections are designed to have different mesa width to obtain a difference in effective index, and thereby to control the spacing of the stop bands. This, in turn, allows beating between two modes and produces selfpulsations. However, two-section laser diodes can also be useful in improving the modulation bandwidth. If beating happens between a main mode and a sufficiently weaker side mode, then the modulation bandwidth is enhanced at the beating frequency, as in [9]. The 3D-view and top-view of the laser structure are shown in Fig. 1 (a, b). During fabrication, the two laser sections are electrically isolated using dry-etching to control the DC current injected in each section independently. Each section is $250 \mu \mathrm{m}$-long. The left and right sections are 4 and $2 \mu \mathrm{m}$ wide, respectively. Adiabatic taper structures are used to couple to the silicon waveguide layer. The right section is modulated while the left laser output is considered. The active region consists of 2 InGaAsP separate confinement heterostructure $(\mathrm{SCH})$ layers of $100 \mathrm{~nm}$ thickness, and 6 InGaAsP quantum wells ( $7 \mathrm{~nm}$ thick, PL wavelength $1550 \mathrm{~nm}$ ) separated by 7 InGaAsP barriers ( $9 \mathrm{~nm}$ thick, PL wavelength of $1550 \mathrm{~nm})$. The thicknesses of the n-InP, p-InP and p-InGaAs are $190 \mathrm{~nm}, 1500 \mathrm{~nm}$ and $220 \mathrm{~nm}$, respectively. The InP epitaxial structure is bonded on a $400 \mathrm{~nm}$-thick Si waveguide (3.5 um wide) that has a $500 \mu \mathrm{m}$-long DFB grating etched 180 $\mathrm{nm}$ deep, with a period of $241 \mathrm{~nm}$ and duty cycle of $50 \%$.

Fig. 2 shows a Focused-Ion-Beam (FIB) cross section image 
of the laser structure near the taper tip. The DVS-BCB bonding layer thickness is less than $10 \mathrm{~nm}$. The laser is encapsulated in $\mathrm{Si}_{3} \mathrm{~N}_{4}$ and $\mathrm{BCB}$ for planarization.
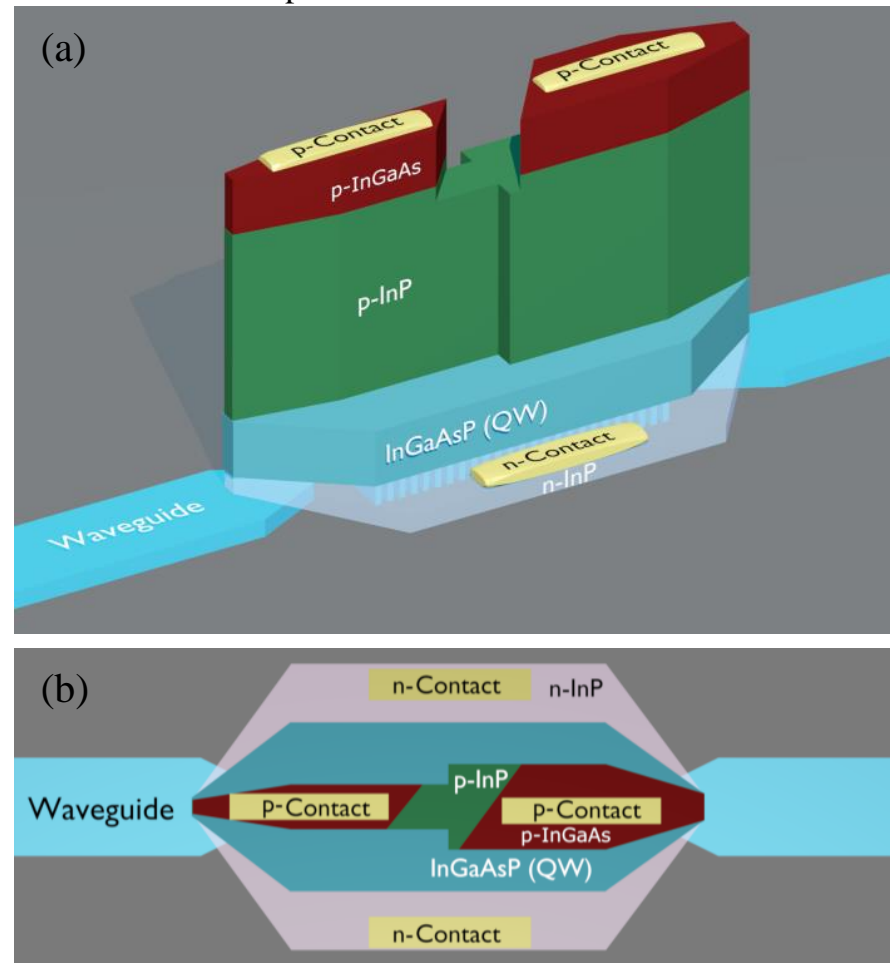

Figure 1. (a) 3D-view and (b) top-view of the fabricated InP-on-Si device showing electrically-isolated laser sections for independent current control.

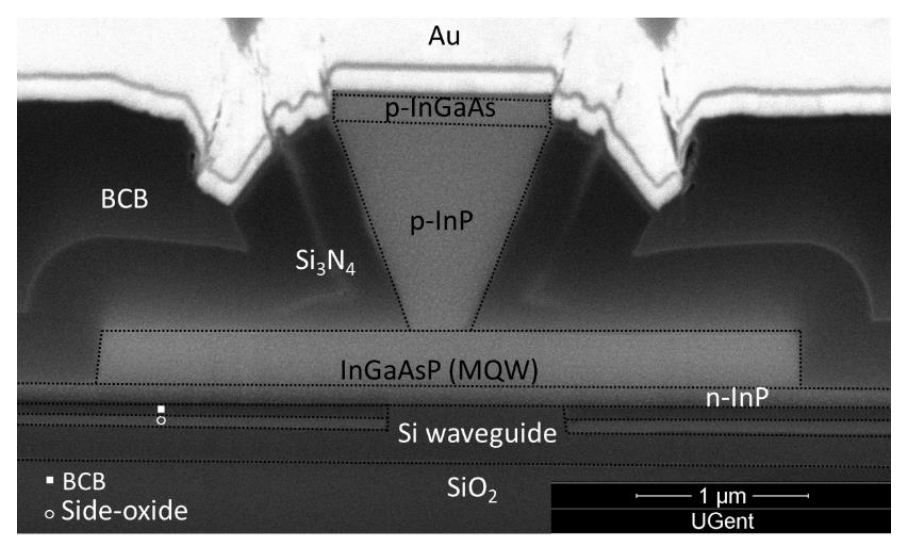

Figure 2. FIB cross-section image of a fabricated device showing the laser structure encapsulated in $\mathrm{Si}_{3} \mathrm{~N}_{4}$ and $\mathrm{BCB}$ on top of a silicon waveguide.

\section{CHARACTERIZATION}

Fig. 3 and 4 show the optical spectrum when the bias currents are $I_{L}=48 \mathrm{~mA}$ and $I_{R}=60 \mathrm{~mA}$, which produces the highest small signal 3-dB modulation bandwidth. As the bonding layer thickness is very small (less than $10 \mathrm{~nm}$ ), the silicon grating coupling is strong. The stop-band of each laser section is not clearly identified, but from characterization it is expected that each section has as stopband of around $5 \mathrm{~nm}$ [3] with the extracted coupling coefficient $\kappa$ being $\sim 150 \mathrm{~cm}^{-1}$. This high coupling coefficient is the source of the multimode behavior observed in the spectrum.

For bandwidth characterization, the laser peak at $1555 \mathrm{~nm}$ is filtered out. A small signal modulation bandwidth close to 25 GHz can be observed in Fig. 5 at $\mathrm{I}_{\mathrm{L}}=46 \mathrm{~mA}$ and $\mathrm{I}_{\mathrm{R}}=60 \mathrm{~mA}$.
Next to the relaxation oscillation frequency around $9 \mathrm{GHz}$ a second resonance around $20 \mathrm{GHz}$ is obtained, which improves the total 3-dB modulation bandwidth by more than a factor of 2. The resonance at $20 \mathrm{GHz}$ results from the beating between the main peak and the side-mode in Fig. 4, which are separated by around $20 \mathrm{GHz}(\approx 0.15 \mathrm{~nm})$. If both modes are of similar strengths, there will be beating around the frequency difference [3]. As a result, the second resonance would be too strong and would lead to too strong distortion. Therefore, the side-mode should be sufficiently weaker than the main mode, for the second resonance to be within the $3-\mathrm{dB}$ small signal modulation range. As shown in [3], it is possible to tune the separation between the peaks, which controls the frequency at which the second resonance occurs, to fine tune the small signal modulation.

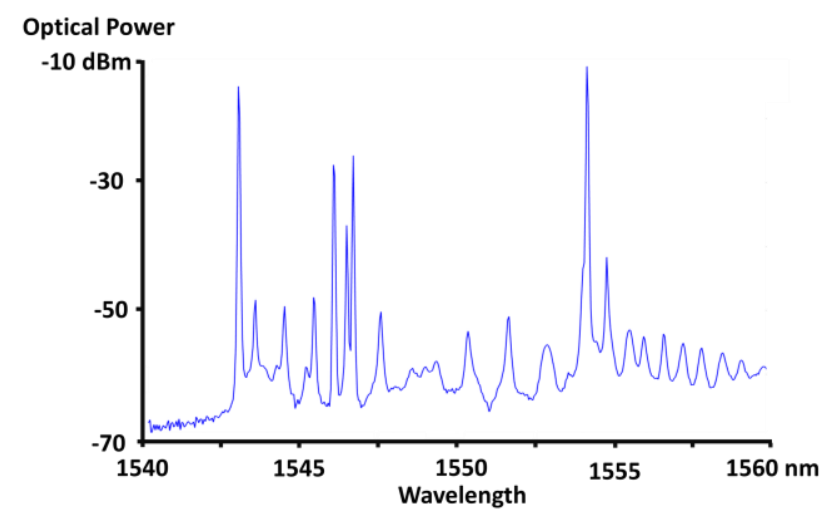

Figure 3. Optical spectrum of the laser at $\mathrm{I}_{\mathrm{L}}=48 \mathrm{~mA}$ and $\mathrm{I}_{\mathrm{R}}=60 \mathrm{~mA}$. The right laser peak is filtered out at the receiver side.

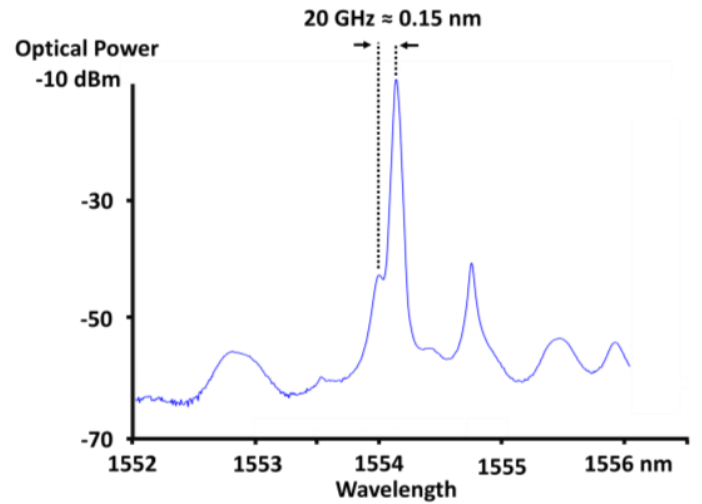

Figure 4 . The filtered laser peak at the received side. The spacing between the main mode and the side mode is about $0.15 \mathrm{~nm}$, which corresponds to $20 \mathrm{GHz}$.

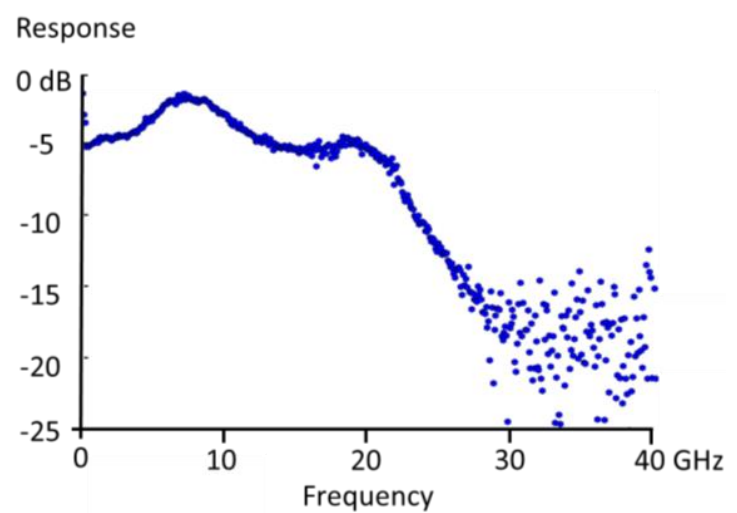

Figure 5. Small signal response showing nearly $25 \mathrm{GHz} 3-\mathrm{dB}$ modulation bandwidth at $I_{L}=46 \mathrm{~mA}$ and $I_{R}=60 \mathrm{~mA}$. 
Data transmission experiments were conducted to investigate the large signal modulation of the laser. Transmission of 45 Gbps non-return-to-zero data that has a pattern length of $2^{7}-1$ shows an open eye-diagram. The voltage swing applied to the right section of the laser is about $2.2 \mathrm{~V}_{\mathrm{pp}}$. Bias conditions of $\mathrm{I}_{\mathrm{L}}=46 \mathrm{~mA}$ and $\mathrm{I}_{\mathrm{R}}=50 \mathrm{~mA}$ were used, resulting in $-5 \mathrm{dBm}$ output power in single mode fiber. The loss from the grating coupler is about $5 \mathrm{~dB}$. An Erbium Doped Fiber Amplifier (EDFA) was used to boost the optical signal and compensate for any losses in the optical link (e.g. grating coupler losses). This is followed by a tunable filter with a bandwidth of $1 \mathrm{~nm}$ to suppress the amplified spontaneous emission from the EDFA. A root-raised-cosine filter with an alpha factor of 0.5 is used to shape the eye diagrams. Fig. 6 shows the eye diagram for a back-to-back configuration, followed by the eye diagram after transmission over a $2-\mathrm{km}$ long Non-Zero Dispersion-Shifted-Fiber (NZ-DSF).

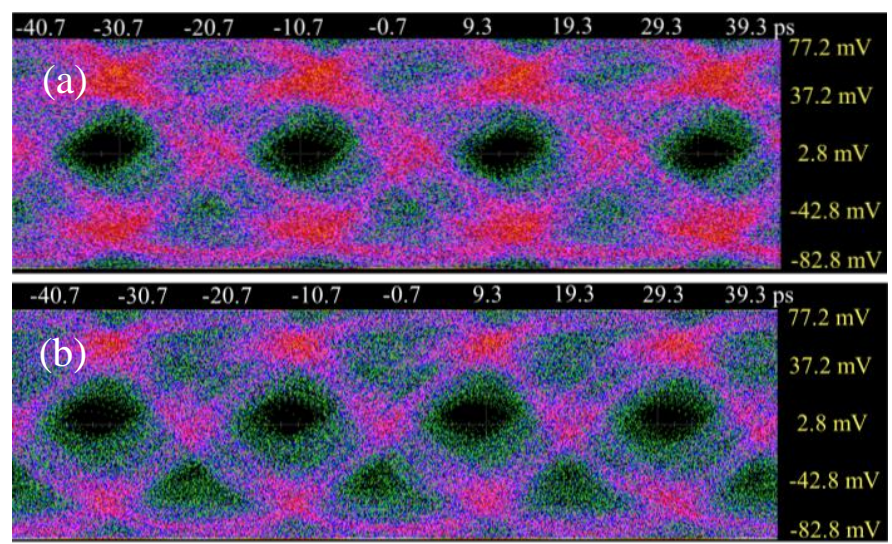

Figure 6. 45 Gbps error-free transmission showing (a) back-to-back and (b) with $2 \mathrm{~km} \mathrm{NZ-DSF}$, for a pattern length of $2^{7}-1$.

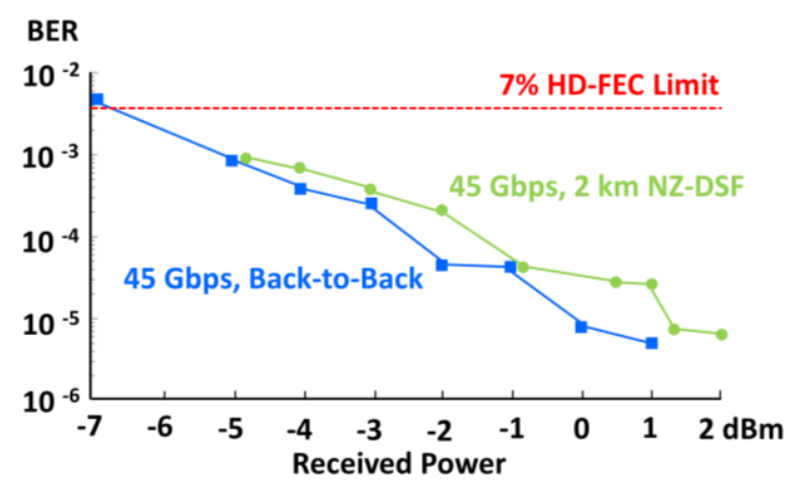

Figure 7. Received power vs. bit-error-rate showing error-free transmission for received power as low as $-7 \mathrm{dBm}$.

The BER vs. received power measurement for this laser is shown in Fig. 7. 45 Gbps transmission with a BER lower than the $7 \%$ Hard Decision (HD) FEC limit is possible for a received signal power above $-7 \mathrm{dBm}$.

\section{CONCLUSIONS}

Two-section InP-on-Si DFB laser diodes with enhanced modulation bandwidth were demonstrated. The modulation bandwidth is estimated to be around $25 \mathrm{GHz}$, allowing data transmission at $45 \mathrm{Gbps}$ both for a back-to-back configuration and for transmission over $2 \mathrm{~km}$ non-zero dispersion-shifted fiber.

\section{ACKNOWLEDGMENT}

The authors acknowledge Methusalem funding of the Flemish government, and the assistance of L. Van Landschoot for FIB images, S. Verstuyft and M. Muneeb for metal deposition, and J. Van Kerrebrouck during high-speed measurement

\section{REFERENCES}

[1] S. Keyvaninia et al., "Ultra-thin DVS-BCB adhesive bonding of III-V wafers, dies and multiple dies to a patterned silicon-on-insulator substrate," Optical Materials Express, 3(1), pp. 35-46, (2012).

[2] S. Dhoore et al., "Demonstration of a discretely tunable IIIV-on-silicon sampled grating DFB laser," IEEE Photonics Technology Letters, 28(21), pp. 2343-2346 (2016).

[3] K. Ma et al., "Demonstration of InP-on-Si Self-Pulsating DFB Laser Diodes for Optical Microwave Generation," IEEE Photonics Journal, 9(4), pp. 1504608, (2017).

[4] A. Abbasi et al., "Direct and electro-absorption modulation of a III-V-on-silicon DFB laser at 56 Gbps," IEEE Journal of Selected Topics in Quantum Electronics, 23(6), pp. 1501307, (2017).

[5] A. Abbasi et al., "2x56 Gbps Electroabsorption Modulated III-V-on-silicon DFB Laser," European Conference on Optical Communication (ECOC), (2017).

[6] G. Roelkens et al., "III-V-on-Silicon Photonic Devices for Optical Communication and Sensing," Photonics (3), pp. 9691004, (2015).

[7] P. Bardella et al., "Design and Analysis of Enhanced Modulation Response in Integrated Coupled Cavities DBR Lasers Using Photon-Photon Resonance," Photonics 3(1), (2016).

[8] T. Numai et al., "High Resonance Frequency in a Coupled Cavity DFB-LD with Phase- Shifted/Uniform Gratings by Photon-Photon Resonance," proceedings in International Conference of Numerical Simulation of Optoelectronic Devices (NUSOD), pp. 35-36, (2017).

[9] Y. Matsui et al., "55 GHz Bandwidth Distributed Reflector Laser," Journal of lightwave technology, 35(3), pp. 397-403, (2017). 